\title{
Red-shifted red/green-type cyanobacteriochrome AM1_1870g3 from the chlorophyll $d$-bearing cyanobacterium Acaryochloris marina
}

Rei Narikawa $^{\text {a,b,1 }}$, Keiji Fushimi ${ }^{\mathrm{a}}, \mathrm{Ni}-\mathrm{Ni}-\mathrm{Win}^{\mathrm{c}}$, and Masahiko Ikeuchi ${ }^{\mathrm{c}, \mathrm{d}}$

${ }^{a}$ Department of Biological Science, Faculty of Science, Shizuoka University, Ohya, Suruga-ku, Shizuoka 422-8529, Japan

${ }^{b}$ Precursory Research for Embryonic Science and Technology, Japan Science and Technology Agency, 4-1-8 Honcho Kawaguchi, Saitama 332-0012 Japan

${ }^{c}$ Department of Life Sciences (Biology), Graduate School of Art and Sciences, University of Tokyo, Komaba, Meguro, Tokyo 153-8902, Japan

${ }^{\mathrm{d}}$ Core Research for Evolutional Science and Technology, Japan Science and Technology Agency, 4-1-8 Honcho Kawaguchi, Saitama 332-0012 Japan

${ }^{1}$ To whom correspondence should be addressed.

Email: narikawa.rei@shizuoka.ac.jp, Tel: +81-54-238-4783 


\begin{abstract}
Cyanobacteriochromes (CBCRs) are diverse photoreceptors that are found only from cyanobacteria and cover wide range of light qualities. CBCRs are divided into two types regarding the chromophore species they contain: phycocyanobilin (PCB) and phycoviolobilin. Red/green-type CBCRs are widely distributed subfamily among the PCB-binding CBCRs and photoconvert between a red-absorbing thermostable form and a green-absorbing metastable form. Our recent study discovered that a red/green-type CBCR, AM1_1557g2, from a cyanobacterium Acaryochloris marina covalently binds not only PCB but also biliverdin (BV). BV-binding AM1_1557g2 photoconverts between a far-red absorbing form and an orange-absorbing form. We report, herein, that another red/green-type CBCR, AM1_1870g3, from the cyanobacterium A. marina also bound both PCB and BV. PCB- and BV-binding ones showed red/green and far-red/orange reversible photoconversions, respectively. Unexpectedly, absorbing wavelengths are 10-20 nm red-shifted compared with those of AM1_1557g2. These red-shifted characteristics may be useful for optogenetic light switches that work in various organisms.
\end{abstract}

Keywords: cyanobacteria; photoreceptor; bilin; cyanobacteriochrome; biliverdin 


\section{Introduction}

Cyanobacteriochromes (CBCRs) are recently identified linear tetrapyrrole-binding photoreceptor family that is found only in cyanobacteria to date and is diverse in their binding chromophores and their sensing light qualities [1]. CBCRs are distant relative of red/far-red light-sensing phytochromes and involved in some photo-acclimation processes such as phototaxis $[2,3,4,5,6,7]$, chromatic acclimation of photosynthetic antennae [8] and light-dependent cell aggregation [9]. CBCRs need only GAF (cGMP-phosphodiesterase/adenylate cyclase/FhlA) domain to ligate chromophores, whereas the phytochromes need a large unit composed of PAS (Per/Arnt/Sim), GAF and PHY (phytochrome-specific) domains for chromophore ligation. CBCRs are divided into two subfamilies regarding the binding chromophore they contain: phycocyanobilin (PCB) and phycoviolobilin. PCB-binding CBCRs sense relatively long wavelength light of blue-to-red region [10,11,12,13,14], whereas phycoviolobilin-binding CBCRs sense rather short wavelength light of ultraviolet-to-green region $[15,16,17,18,19,20]$.

Among the PCB-binding CBCRs, red/green-type CBCRs are widely distributed and considerably analyzed so far [10,14,21]. The red/green-type CBCRs covalently bind PCB and reversibly photoconvert between a red-absorbing thermostable form $(\mathrm{Pr})$ and a green-absorbing metastable form $(\mathrm{Pg})$. The red-absorbing form is quite similar to that of the phytochromes, whereas the green-absorbing form is clearly different from the far-red absorbing form of the phytochromes. Although structural and spectral studies suggest that the unique blue-shift of the Pg form is due to ring D twist and/or chromophore hydration, most of their color tuning mechanisms remain still unclear [22,23,24,25,26,27,28]. 
Recently, we identified that a red/green-type CBCR AM1_1557g2 from the chlorophyll $d$-bearing Acaryochloris marina covalently binds not only PCB but also BV [29]. BV-binding AM1_1557g2 shows reversible photoconversion between a far-red absorbing thermostable form (Pfr) and an orange-absorbing metastable form (Po). The Pfr form fluoresces with a maximum at $730 \mathrm{~nm}$. Because A. marina mainly utilizes chlorophyll $d$ as a photosynthetic pigment that is red-shifted compared with chlorophyll $a$, the BV-binding ability of AM1_1557g2 may be a physiologically relevant feature of A. marina.

In this study, we identified another red/green-type CBCR AM1_1870g3 from A. marina that also covalently binds both PCB and BV. Both PCB- and BV-binding ones absorb 10-20 nm red-shifted light qualities compared with those of the other red/green-type CBCRs. In this context, potentials of this red-shifted CBCR as the optogenetic switch are discussed. 


\section{Methods}

2.1. In silico Characterization of AM1_1870. The domain composition of AM1_1870 was predicted using SMART program on the web site (http://smart.embl-heidelberg.de/) [30]. Alignment and phylogenetic clustering of CBCR and phytochrome GAF domains were performed by CLUSTAL_X [31]. The phylogenetic tree was drawn by Dendroscope [32].

2.2. Plasmid Construction. The nucleotide sequence of AM1_1870g3 was cloned into pET28a (Novagen) using the In-Fusion HD Cloning kit (TaKaRa). The DNA fragment corresponding to AM1_1870g3 was PCR amplified using the synthetic primers 5'-CGCGGCAGCCATATGGTCCAGACCCTCAATACC-3' (forward primer) and 5'-CTCGAATTCGGATCCTCAAACGGCGAGCTGTTGCGC-3' (reverse primer), genomic DNA from A. marina MBIC11017, and PrimeSTAR Max DNA polymerase. pET28a was PCR amplified using the synthetic primers 5'-CATATGGCTGCCGCGCGG-3' (forward primer) and 5'-GGATCCGAATTCGAGCTC-3' (reverse primer), pET28a, and PrimeSTAR Max DNA polymerase. A plasmid expressing AM1_1870g3 (pET28a_AM1_1870g3) was then constructed with the TaKaRa in-fusion system reagents. The sequence of the gene encoding AM1_1870g3 was verified by DNA sequencing.

2.3. Expression and Purification of His-tagged AM1_1870g3. E. coli C41 (Novagen) carrying pKT270 or pKT271 [33] was used for AM1_1870g3 expression. Each culture was incubated at $37^{\circ} \mathrm{C}$ for $2.5 \mathrm{~h}$ in $1 \mathrm{~L}$ of Luria-Bertani medium, $20 \mu \mathrm{g} \mathrm{ml} \mathrm{l}^{-1}$ kanamycin, 
and $20 \mu \mathrm{g} \quad \mathrm{ml}^{-1}$ chloramphenicol, followed by addition of isopropyl-thio- $\beta$-D-galactopyranoside (final concentration, $0.1 \mathrm{mM}$ ). Cells were then cultured at $18^{\circ} \mathrm{C}$ overnight, after which they were harvested by centrifugation, frozen at $-80^{\circ} \mathrm{C}$, thawed at $4^{\circ} \mathrm{C}$, and suspended in $50 \mathrm{ml}$ of Buffer A $(20 \mathrm{mM}$ HEPES-NaOH, pH 7.5, $100 \mathrm{mM} \mathrm{NaCl}, 10 \%$ (w/v) glycerol). Cells were disrupted by three passages through an Emulsiflex C5 high-pressure homogenizer at 12,000 psi (Avestin). The cell extract was then centrifuged at $109,200 \times g$ for 30 min at $4^{\circ} \mathrm{C}$. Each supernatant was individually passed through a nickel-affinity His-trap chelating column (GE Healthcare). After washing the column with Buffer A containing $30 \mathrm{mM}$ imidazole, the binding proteins were eluted using a step gradient of 50,100, and $200 \mathrm{mM}$ imidazole in Buffer A. His-tagged proteins were mostly eluted in the $200-\mathrm{mM}$ imidazole fraction, which was studied after removal of imidazole by dialysis against Buffer A.

2.4. SDS-PAGE and Zn-induced Fluorescence Assay. Proteins in $2 \%$ (w/v) lithium dodecylsulfate, $60 \mathrm{mM}$ DTT, $60 \mathrm{mM}$ Tris-HCl, $\mathrm{pH} 8.0$ were subjected to SDS-PAGE (15\% (w/v) acrylamide), followed by staining with Coomassie Brilliant Blue (CBB) R-250. For the Zn-induced fluorescence assay, after SDS-PAGE, the gel was soaked in $20 \mu \mathrm{M}$ zinc acetate at room temperature for $30 \mathrm{~min}$ [34]. Then, fluorescence was visualized through a $605 \mathrm{~nm}$ filter upon excitation at $532 \mathrm{~nm}$ (FMBIO II; Takara).

2.5. Spectroscopy. Ultraviolet and visible absorption spectra of the proteins were recorded using a Shimadzu UV-2600 spectrophotometer at room temperature. Monochromic light of various wavelengths was generated using a variable wavelength light source (Opto-Spectrum Generator, Hamamatsu Photonics, Inc.). After denaturing 
the proteins in $8 \mathrm{M}$ urea, $\mathrm{pH} 2.0$, their absorption spectra were recorded. Then, the protein samples were irradiated with white light for $3 \mathrm{~min}$, and absorption spectra were again recorded. 


\section{Results and Discussion}

\subsection{Sequence characteristics of AM1_1870g3}

AM1_1870 is a multi-domain two-component signal-transduction protein of 1514 amino acid residues. AM1_1870 is composed of four GAF domains, one His kinase (HK) domain, two response regulator (RR) domains and one phospho-accepting (HPT) domain (Fig. 1A). Third GAF domain (AM1_1870g3, 512-668 residues) belongs to the red/green-type CBCR GAF domain according to our clustering analysis, whereas first and fourth GAF domains belong to phytochrome-type and blue/green-type, respectively (Fig. 1B). These three GAF domains retain residues that are important for chromophore ligation and photoconversion. In this context, AM1_1870 is a quite unusual protein that contains three distinctive light sensing domains within one protein.

\subsection{Photoconversion of AM1_1870g3-PCB and AM1_1870g3-BV}

AM1_1870g3 expressed in a PCB-producing E. coli was purified into near homogeneity (Fig. 2A, CBB). PCB covalently bound to AM1_1870g3 judging from Zn-dependent fluorescence assay (Fig. 2A, +Zn). AM1_1870g3-PCB showed reversible photoconversion between a red-absorbing (Pr) form peaking at $665 \mathrm{~nm}$ and a green-absorbing (Pg) form peaking at $561 \mathrm{~nm}$ (Fig. 2B). In the previous reports of the other red/green-type CBCRs, NpR4776g3-PCB showed most red-shifted characteristics: Pr form peaking at $656 \mathrm{~nm}$ and $\mathrm{Pg}$ form peaking at $558 \mathrm{~nm}$ [21]. In this context, AM1_1870g3 is most red-shifted CBCR among the known red/green-type CBCRs to date. 
AM1_1870g3 expressed in a BV-producing E. coli was purified with some contaminated proteins (Fig. 2D, CBB). The main band stained by CBB corresponded to the fluorescent band in the Zn-dependent fluorescent assay, indicating that BV covalently bound to AM1_1870g3 (Fig. 2D, +Zn). Dark-incubated AM1_1870g3-BV had an absorbance peaking at $706 \mathrm{~nm}$ (Fig. 2E, solid line). Because AM1_1870g3-BV showed quite fast dark reversion (Fig. 2F), we measured a spectrum of AM1_1870g3 while irradiating with far-red light (Fig. 2E, dashed line). The obtained spectrum had an absorbance peaking at $641 \mathrm{~nm}$. This is a reversible conversion between a far red-absorbing (Pfr) form and an orange-absorbing (Po) form (Fig. 2E). The absorbance maxima of AM1_1870g3-BV Pfr and Po are 41- and 80-nm red-shifted compared with those of AM1_1870g3-PCB Pr and Pg, respectively. Both forms of PCB- and BV-binding ones are 10-20 nm red-shifted compared with those of AM1_1557g2 (Fig. 3). The (Pfr - Po) spectrum has maxima at 713 and $375 \mathrm{~nm}$, and a minimum at $616 \mathrm{~nm}$ (Fig. 3B), whereas the $(\operatorname{Pr}-\mathrm{Pg})$ spectrum has maxima at 665 and $353 \mathrm{~nm}$, and a minimum at $559 \mathrm{~nm}$ (Fig. 3A). Judging from the ratio of the maximum aromatic residues absorbance (at $280 \mathrm{~nm}$ ) to chromophore absorbance (at $706 \mathrm{~nm}$ for AM1_1870g3-BV and 665 nm for AM1_1870g3-PCB), the binding efficiency of BV to AM1_1870g3 is lower than that of PCB (Fig. 2B and 2E).

\subsection{Chromophore Species, Their Configurations and Dark Reversion Kinetics}

To identify the chromophore species and their configurations, spectra were obtained for acid-denatured AM1_1870g3-PCB and -BV. Absorption maxima of denatured AM1_1870g3-PCB Pr and Pg were observed at 664 and 594 nm, respectively (Fig. 4A and B, solid lines). Absorption maxima of denatured 
AM1_1870g3-BV Pfr and Po were observed at $\sim 700$ and $\sim 620 \mathrm{~nm}$, respectively (Fig. 4C and D, solid lines). Irradiation of denatured Pg and Po with white light resulted in red shift of the absorption spectra (Fig. 4B and D, dashed lines), whereas irradiation of denatured Pr and Pfr with white light resulted in no appreciable spectral change (Fig. 4A and $\mathrm{C}$, dashed lines). These results showed that $\mathrm{Pr}$ and $\mathrm{Pfr}$ forms bound 15Z-chromophores, whereas Pg and Po forms bound 15E-chromophores.

We measured dark reversion kinetics of the thermostable states ( $\operatorname{Pr}$ of AM1_1870g3-PCB and Pfr of AM1_1870g3-BV) at room temperature. AM1_1870g3-PCB showed relatively slow dark reversion with half-life of 29 minutes (Fig. 2C), whereas AM1_1870g3-BV showed quick dark reversion with half-life of 30 seconds (Fig. 2F). It is of note that these dark reversions are largely faster than those of AM1_1557g2 (93 hours for AM1_1157g2-PCB and 1 hour for AM1_1557g2-BV) and that reversion of the BV-binding one is 60-times faster than that of the PCB-binding one, which is consistent with the case for AM1_1557g2 [29]. This indicates that common structural arrangement that facilitates dark reversion of $\mathrm{BV}$-binding ones but not PCB-binding ones underlies the chromophore-binding pockets of AM1_1870g3 and AM1_1557g2.

\subsection{BV-binding and red-shifted features}

Although AM1_1870g3 is able to covalently ligate BV, its binding efficiency to BV is lower than that of AM1_1557g2 [29]. On the other hand, AnPixJg2 is not able to ligate BV [10]. In this context, AM1_1870g3 can be considered to have a moderate ability to bind BV among the red/green-type CBCRs. In the case of AM1_1557g2, site-directed mutagenesis suggests that Leu337 near the ring D is a major factor for 
potential to bind BV [29]. AM1_1870g3 possesses His residue (His633) instead of Leu, whereas the canonical red/green-type CBCRs including AnPixJg2 possess Asn residue (Asn354 in AnPixJg2). Variation of the amino acid residues at this position may have a correlation with binding selectivity to BV. Future study to introduce mutation in the chromophore-binding pocket of AM1_1870g3 may improve its binding efficiency to BV.

Both forms of PCB- and BV-binding AM1_1870g3 are 10-20 nm red-shifted compared with those of AM1_1557g2. There may be unique residues near the chromophore crucial for the red-shifted feature of AM1_1870g3. Although we compared residues near the chromophore of AM1_1870g3 with the other red/green-type CBCRs based on the AnPixJg2 structure, we failed to detect obvious candidate residues. Residues distant from the chromophore may indirectly affect interaction between the chromophore and AM1_1870g3 to result in the red shift.

\subsection{Prospect for development of light switches that work in various organisms}

The red-shifted feature of AM1_1870g3 may be useful for application to the photosynthetic organisms. Photosynthesis is driven mainly by blue and red light qualities that are efficiently absorbed by chlorophylls, whereas green and far-red light qualities are not so utilized for photosynthesis. In this context, in order to regulate production of useful substances such as biofuels and chemicals without loss of photosynthetic productivity, far-red/green reversible feature is suitable for optogenetic light switches that work in the photosynthetic organisms. AM1_1870g3-BV shows far-red/orange reversible photoconversion and its Pfr form is approximately $15 \mathrm{~nm}$ red-shifted compared with that of AM1_1557g2. Thus, the Pfr form of AM1_1870g3 
well escapes from the chlorophyll $a$ absorbance peaking at $\sim 675 \mathrm{~nm}$ in the red region. If we succeed in blue shift of Po form to absorb green light, such photoreceptor can be well utilized as an optogenetic light switch in the photosynthetic organisms.

We recently identified another CBCR GAF domain, AM1_1186g2, that covalently binds PCB and shows reversible photoconversion between a red-absorbing thermostable form and a blue-absorbing metastable form [12]. Although the AM1_1186g2 also belongs to the red/green-type CBCRs based on the primary sequence, its metastable form is quite blue shifted compared with that of the red/green-type CBCRs. AM1_1186g2 has a Cys residue near the chromophore-binding pocket and this Cys residue transiently ligates to the chromophore and resultantly the quite blue-shifted $\mathrm{Pb}$ form is generated. Notably, this Cys residue does not correspond to those of the blue/green-type CBCRs that also forms transient covalent bond with the chromophore on the primary sequence. Introduction of a Cys residue at certain position similar to the second Cys residue of AM1_1186g2 may result in transient covalent bond formation and consequent blue shift of the Po form.

Because visible light but not far-red light is largely absorbed by melanin and heme in the mammal tissues, far red-responsive proteins are also useful for application to the mammal deep tissues. Further, mammal cells are known to intrinsically possess biliverdin as a degradation product from heme and the intrinsic biliverdin has been reported to serve as a chromophore for an extrinsic photoreceptor [35]. Because the Pfr form of AM1_1870g3-BV absorbs most red-shifted light quality peaking at $706 \mathrm{~nm}$ among the known CBCRs, AM1_1870g3-BV may be a promising developmental platform for the optogenetic tool in the mammal tissues. 


\section{Acknowledgements}

This work was supported by Precursory Research for Embryonic Science and Technology, Japan Science and Technology Agency, 4-1-8 Honcho Kawaguchi, Saitama 332-0012 (to R.N.) and Grants-in-Aid for Young Scientists (to R.N.). 


\section{References}

[1] M. Ikeuchi, T. Ishizuka, Cyanobacteriochromes: a new superfamily of tetrapyrrole-binding photoreceptors in cyanobacteria, Photochem. Photobiol. Sci. 7 (2008) 1159-1167.

[2] E.L. Campbell, K.D. Hagen, R. Chen, D.D. Risser, D.P. Ferreira, J.C. Meeks, Genetic analysis reveals the identity of the photoreceptor for phototaxis in hormogonium filaments of Nostoc punctiforme, J. Bacteriol. 197 (2015) 782-791.

[3] P. Savakis, S. De Causmaecker, V. Angerer, U. Ruppert, K. Anders, L.O. Essen, A. Wilde, Light-induced alteration of c-di-GMP level controls motility of Synechocystis sp. PCC 6803, Mol. Microbiol. 85 (2012) 239-251.

[4] J.Y. Song, H.S. Cho, J.I. Cho, J.S. Jeon, J.C. Lagarias, Y.I. Park, Near-UV cyanobacteriochrome signaling system elicits negative phototaxis in the cyanobacterium Synechocystis sp. PCC 6803, Proc. Natl. Acad. Sci. U. S. A. 108 (2011) 10780-10785.

[5] R. Narikawa, F. Suzuki, S. Yoshihara, S. Higashi, M. Watanabe, M. Ikeuchi, Novel photosensory two-component system (PixA-NixB-NixC) involved in the regulation of positive and negative phototaxis of cyanobacterium Synechocystis sp. PCC 6803, Plant Cell Physiol. 52 (2011) 2214-2224.

[6] Y.J. Moon, S.Y. Kim, K.H. Jung, J.S. Choi, Y.M. Park, Y.H. Chung, Cyanobacterial phytochrome Cph2 is a negative regulator in phototaxis toward UV-A, FEBS Lett. 585 (2011) 335-340.

[7] S. Yoshihara, F. Suzuki, H. Fujita, X.X. Geng, M. Ikeuchi, Novel putative photoreceptor and regulatory genes required for the positive phototactic movement of the unicellular motile cyanobacterium Synechocystis sp. PCC 6803, Plant Cell Physiol. 41 (2000) 1299-1304.

[8] Y. Hirose, R. Narikawa, M. Katayama, M. Ikeuchi, Cyanobacteriochrome CcaS regulates phycoerythrin accumulation in Nostoc punctiforme, a group II chromatic adapter, Proc. Natl. Acad. Sci. U. S. A. 107 (2010) 8854-8859.

[9] G. Enomoto, R. Nomura, T. Shimada, N.N. Win, R. Narikawa, M. Ikeuchi, Cyanobacteriochrome SesA is a diguanylate cyclase that induces cell aggregation in Thermosynechococcus, J. Biol. Chem. 289 (2014) 24801-24809.

[10] R. Narikawa, Y. Fukushima, T. Ishizuka, S. Itoh, M. Ikeuchi, A novel photoactive GAF domain of cyanobacteriochrome AnPixJ that shows reversible green/red photoconversion, J. Mol. Biol. 380 (2008) 844-855.

[11] Y. Hirose, T. Shimada, R. Narikawa, M. Katayama, M. Ikeuchi, Cyanobacteriochrome CcaS is the green light receptor that induces the expression of phycobilisome linker protein., Proc. Natl. Acad. Sci. U. S. A. 105 (2008) 9528-9533.

[12] R. Narikawa, G. Enomoto, W. Ni Ni, K. Fushimi, M. Ikeuchi, A new type 
of dual-Cys cyanobacteriochrome GAF domain found in cyanobacterium Acaryochloris marina, which has an unusual red/blue reversible photoconversion cycle, Biochemistry 53 (2014) 5051-5059.

[13] N.C. Rockwell, S.S. Martin, K. Feoktistova, J.C. Lagarias, Diverse two-cysteine photocycles in phytochromes and cyanobacteriochromes, Proc. Natl. Acad. Sci. U. S. A. 108 (2011) 11854-11859.

[14] Y. Chen, J. Zhang, J. Luo, J.M. Tu, X.L. Zeng, J. Xie, M. Zhou, J.Q. Zhao, H. Scheer, K.H. Zhao, Photophysical diversity of two novel cyanobacteriochromes with phycocyanobilin chromophores: photochemistry and dark reversion kinetics, FEBS J. 279 (2012) 40-54.

[15] N.C. Rockwell, S.S. Martin, A.G. Gulevich, J.C. Lagarias, Phycoviolobilin formation and spectral tuning in the DXCF cyanobacteriochrome subfamily, Biochemistry 51 (2012) 1449-1463.

[16] Q. Ma, H.H. Hua, Y. Chen, B.B. Liu, A.L. Kramer, H. Scheer, K.H. Zhao, M. Zhou, A rising tide of blue-absorbing biliprotein photoreceptors: characterization of seven such bilin-binding GAF domains in Nostoc sp. PCC7120, FEBS J. 279 (2012) 4095-4108.

[17] N.C. Rockwell, S.L. Njuguna, L. Roberts, E. Castillo, V.L. Parson, S. Dwojak, J.C. Lagarias, S.C. Spiller, A second conserved GAF domain cysteine is required for the blue/green photoreversibility of cyanobacteriochrome Tlr0924 from Thermosynechococcus elongatus, Biochemistry 47 (2008) 7304-7316.

[18] G. Enomoto, Y. Hirose, R. Narikawa, M. Ikeuchi, Thiol-based photocycle of the blue and teal light-sensing cyanobacteriochrome Tlr1999, Biochemistry 51 (2012) 3050-3058.

[19] S. Yoshihara, M. Katayama, X. Geng, M. Ikeuchi, Cyanobacterial phytochrome-like PixJ1 holoprotein shows novel reversible photoconversion between blue- and green-absorbing forms, Plant Cell Physiol. 45 (2004) 1729-1737.

[20] T. Ishizuka, T. Shimada, K. Okajima, S. Yoshihara, Y. Ochiai, M. Katayama, M. Ikeuchi, Characterization of cyanobacteriochrome TePixJ from a thermophilic cyanobacterium Thermosynechococcus elongatus strain BP-1, Plant Cell Physiol. 47 (2006) 1251-1261.

[21] N.C. Rockwell, S.S. Martin, J.C. Lagarias, Red/Green cyanobacteriochromes: sensors of color and power, Biochemistry 51 (2012) 9667-9677.

[22] N.C. Rockwell, S.S. Martin, A.G. Gulevich, J.C. Lagarias, Conserved phenylalanine residues are required for blue-shifting of cyanobacteriochrome photoproducts, Biochemistry 53 (2014) 3118-3130.

[23] F. Velazquez Escobar, T. Utesch, R. Narikawa, M. Ikeuchi, M.A. Mroginski, W. Gartner, P. Hildebrandt, Photoconversion mechanism of the second GAF domain of cyanobacteriochrome AnPixJ and the cofactor structure of its green-absorbing state, Biochemistry 52 (2013) 4871-4880. 
[24] R. Narikawa, T. Ishizuka, N. Muraki, T. Shiba, G. Kurisu, M. Ikeuchi, Structures of cyanobacteriochromes from phototaxis regulators AnPixJ and TePixJ reveal general and specific photoconversion mechanism, Proc. Natl. Acad. Sci. U. S. A. 110 (2013) 918-923.

[25] Y. Fukushima, M. Iwaki, R. Narikawa, M. Ikeuchi, Y. Tomita, S. Itoh, Photoconversion mechanism of a green/red photosensory cyanobacteriochrome AnPixJ: time-resolved optical spectroscopy and FTIR analysis of the AnPixJ-GAF2 domain, Biochemistry 50 (2011) 6328-6339.

[26] P.W. Kim, L.H. Freer, N.C. Rockwell, S.S. Martin, J.C. Lagarias, D.S. Larsen, Second-chance forward isomerization dynamics of the red/green cyanobacteriochrome NpR6012g4 from Nostoc punctiforme, J. Am. Chem. Soc. 134 (2011) 130-133.

[27] P.W. Kim, L.H. Freer, N.C. Rockwell, S.S. Martin, J.C. Lagarias, D.S. Larsen, Femtosecond photodynamics of the red/green cyanobacteriochrome NpR6012g4 from Nostoc punctiforme. 1. Forward dynamics, Biochemistry 51 (2011) 608-618.

[28] P.W. Kim, L.H. Freer, N.C. Rockwell, S.S. Martin, J.C. Lagarias, D.S. Larsen, Femtosecond photodynamics of the red/green cyanobacteriochrome NpR6012g4 from Nostoc punctiforme. 2. reverse dynamics, Biochemistry 51 (2011) 619-630.

[29] R. Narikawa, T. Nakajima, Y. Aono, K. Fushimi, G. Enomoto, W. Ni Ni, S. Itoh, M. Sato, M. Ikeuchi, A biliverdin-binding cyanobacteriochrome from the chlorophyll $d$-bearing cyanobacterium Acaryochloris marina, Sci. Rep. 5 (2015) 7950.

[30] J. Schultz, F. Milpetz, P. Bork, C.P. Ponting, SMART, a simple modular architecture research tool: identification of signaling domains, Proc. Natl. Acad. Sci. U. S. A. 95 (1998) 5857-5864.

[31] J.D. Thompson, T.J. Gibson, F. Plewniak, F. Jeanmougin, D.G. Higgins, The CLUSTAL_X windows interface: flexible strategies for multiple sequence alignment aided by quality analysis tools, Nucleic Acids Res. 25 (1997) 4876-4882.

[32] D.H. Huson, D.C. Richter, C. Rausch, T. Dezulian, M. Franz, R. Rupp, Dendroscope: An interactive viewer for large phylogenetic trees, BMC bioinformatics 8 (2007) 460 .

[33] K. Mukougawa, H. Kanamoto, T. Kobayashi, A. Yokota, T. Kohchi, Metabolic engineering to produce phytochromes with phytochromobilin, phycocyanobilin, or phycoerythrobilin chromophore in Escherichia coli, FEBS Lett. 580 (2006) 1333-1338.

[34] T.R. Berkelman, J.C. Lagarias, Visualization of bilin-linked peptides and proteins in polyacrylamide gels, Anal. Biochem. 156 (1986) 194-201.

[35] C. Gasser, S. Taiber, C.M. Yeh, C.H. Wittig, P. Hegemann, S. Ryu, F. Wunder, A. Moglich, Engineering of a red-light-activated human cAMP/cGMP-specific phosphodiesterase, Proc. Natl. Acad. Sci. U. S. A. 111 (2014) 8803-8808. 


\section{Figure legends}

Fig. 1. AM1_1870. (A) Domain architecture of AM1_1870 according to a SMART motif analysis. (B) Cluster analysis of red/green-, green/red- and blue/green-type CBCR and phytochrome-type GAF domains. The position of AM1_1870g3 on the tree was highlighted in red.

Fig. 2. PCB-binding and BV-binding AM1_1870g3. (A) His-tagged AM1_1870g3-PCB isolated from PCB-producing E. coli. CBB-stained gel (CBB) and in-gel Zn-dependent fluorescence assay (+Zn). (B) Absorption spectra of Pr (solid line) and Pg (dashed line) forms of AM1_1870g3-PCB. (C) Dark reversion kinetics of the Pr form of AM1_1870g3-PCB at room temperature. Absorbance at $665 \mathrm{~nm}$ was monitored. (D) His-tagged AM1_1870g3-BV isolated from BV-producing E. coli. CBB-stained gel (CBB) and in-gel Zn-dependent fluorescence assay (+Zn). (E) Absorption spectra of Pfr (solid line) and Po (dashed line) forms of AM1_1870g3-BV. Inset is focused on the chromophore absorbance. (F) Dark reversion kinetics of the Pfr form of AM1_1870g3-BV at room temperature. Absorbance at $710 \mathrm{~nm}$ was monitored.

Fig. 3. Comparison with AM1_1557g2. (A) Pr-minus-Pg difference spectra of AM1_1870g3-PCB (solid line) in comparison with that of AM1_1557g2-PCB (dashed line). (B) Pfr-minus-Po difference spectra of AM1_1870g3-BV (solid line) in comparison with that of AM1_1557g2-BV (dashed line). 
Fig. 4. Chromophore species and configurations. (A) Absorbance spectra of denatured Pr form of AM1_1870g3-PCB. Dark form (solid line) and illuminated one (dashed line). (B) Absorbance spectra of denatured Pg form of AM1_1870g3-PCB. Dark form (solid line) and illuminated one (dashed line). (C) Absorbance spectra of denatured Pfr form of AM1_1870g3-BV. Dark form (solid line) and illuminated one (dashed line). (D) Absorbance spectra of denatured Po form of AM1_1870g3-BV. Dark form (solid line) and illuminated one (dashed line). 
A

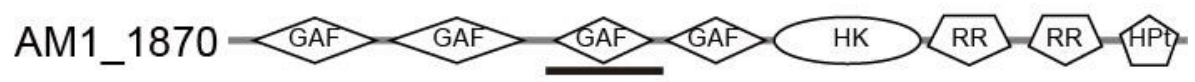

B

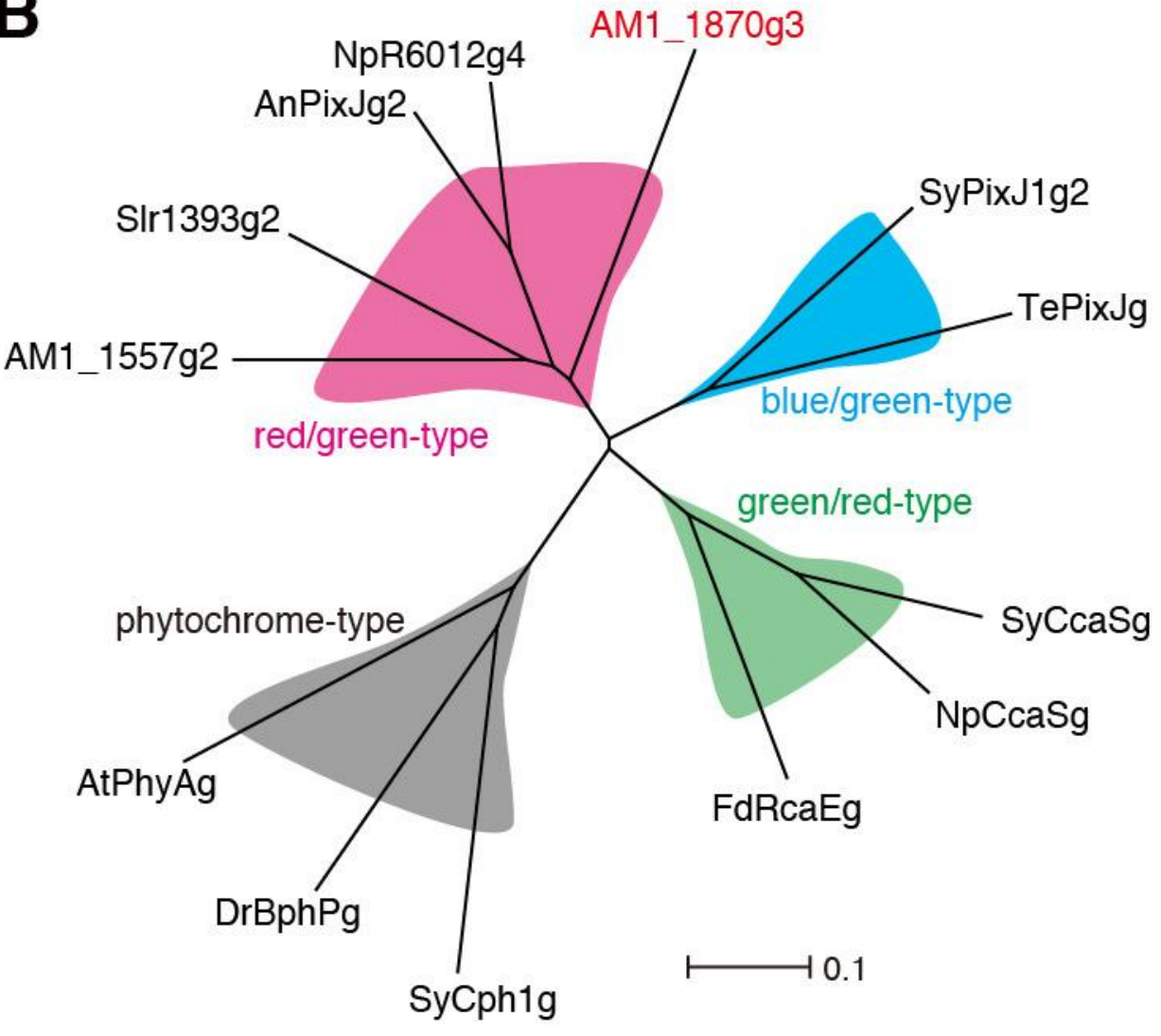

Figure 1 

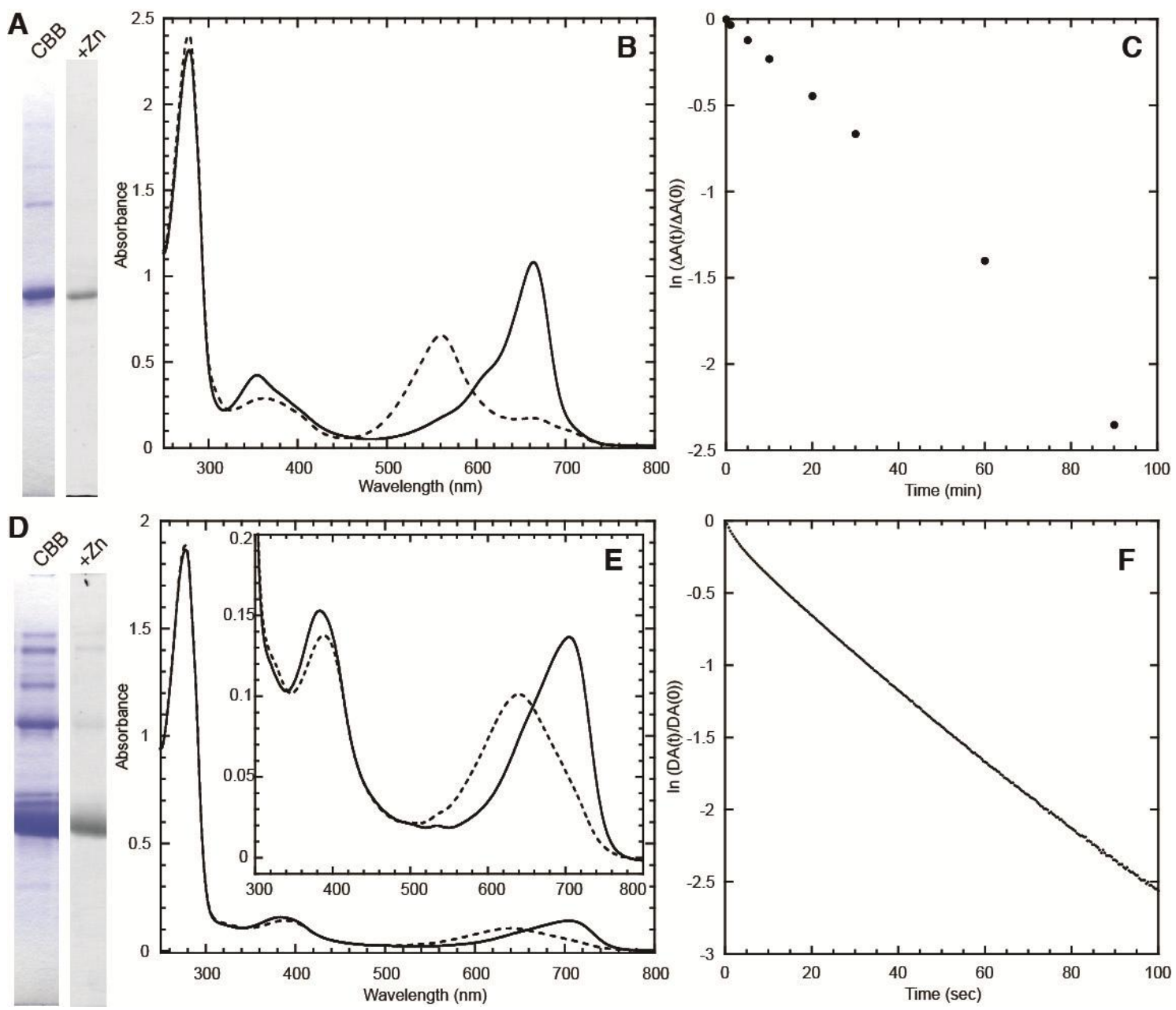

Figure 2 

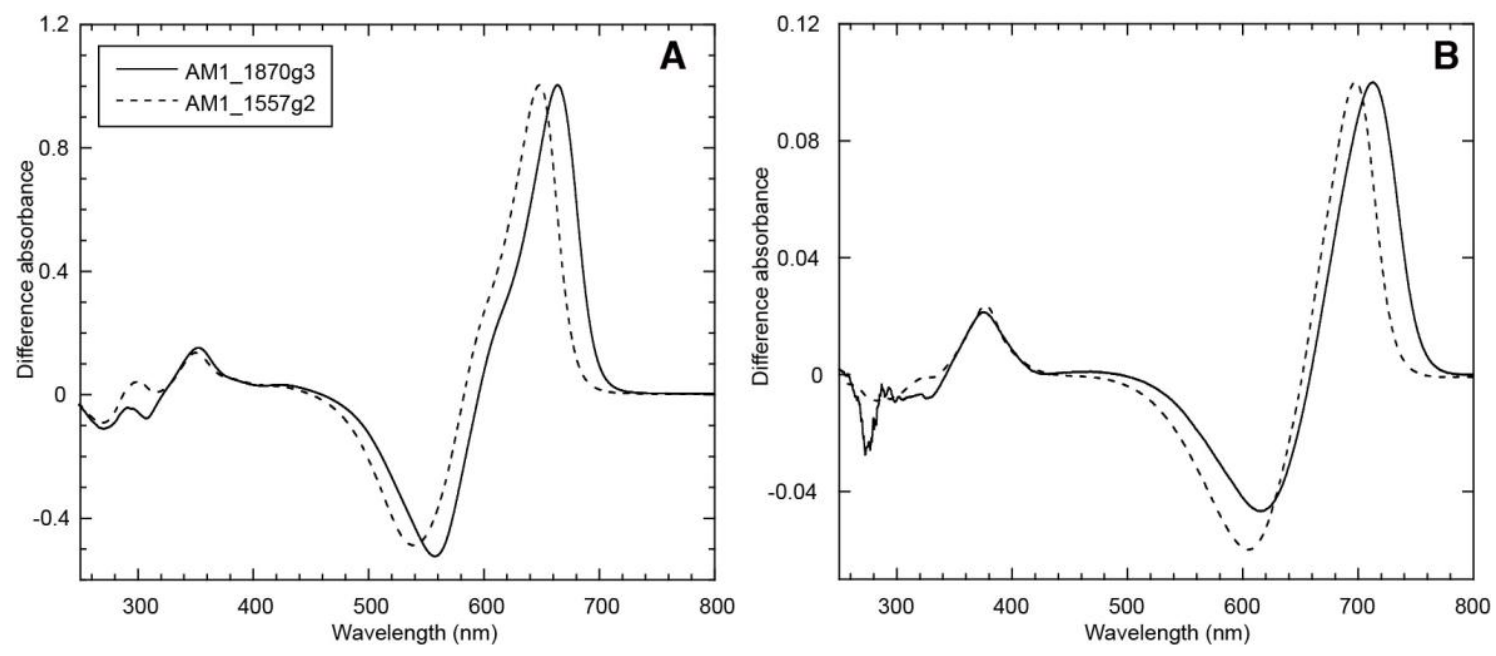

Figure 3 


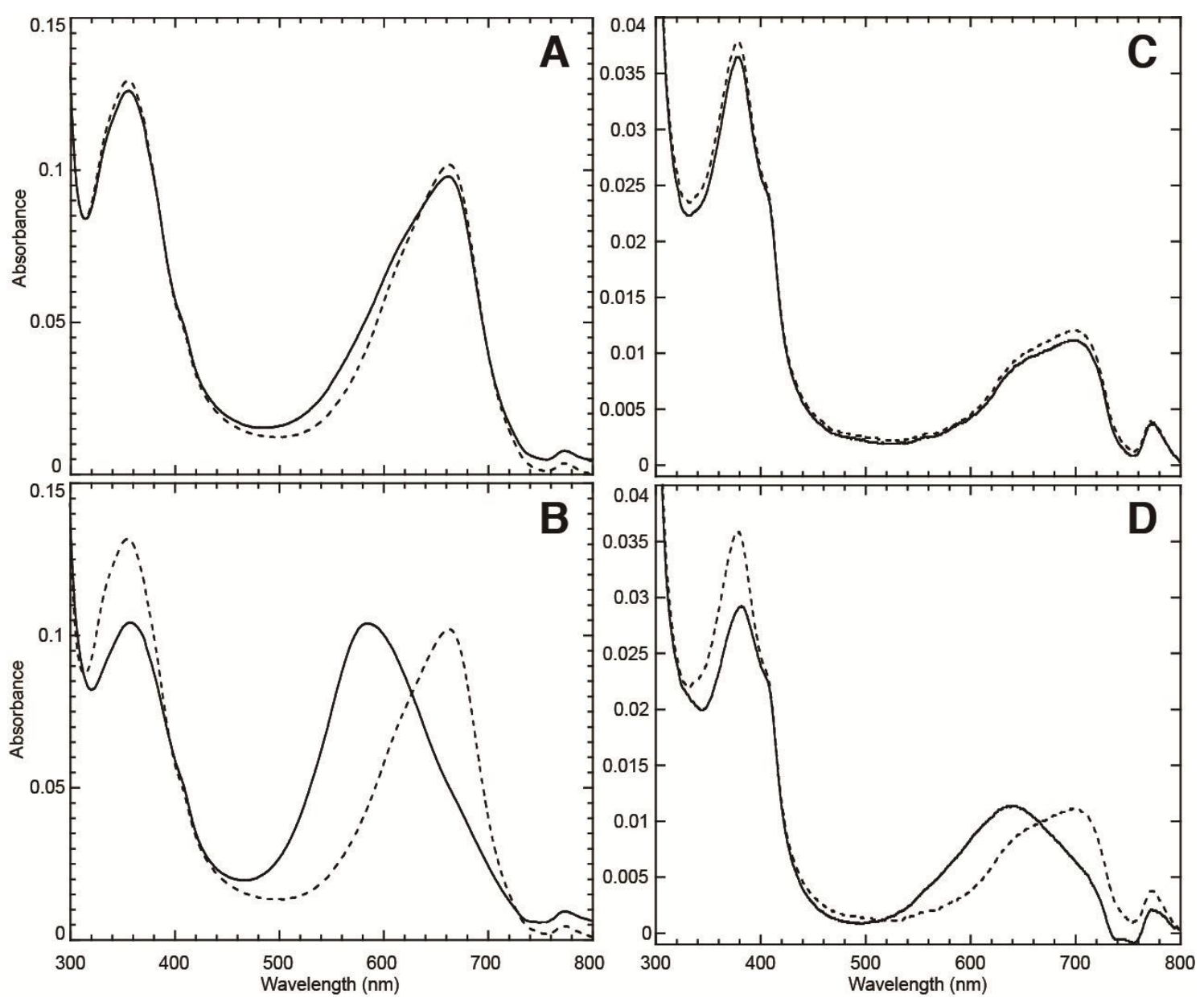

Figure 4 